\title{
Are Women Affinity Groups Enough to Solve the Retention Problem of Women in the IT Workforce?
}

\author{
Hala Annabi \\ The Information School, University of Washington \\ hpannabi@uw.edu
}

\begin{abstract}
Women Affinity Groups (WAGs) are the most prevalent diversity and inclusion (D\&I) intervention method utilized in the Information Technology industry (IT) to improve the participation of women. Ninety three percent of organizations surveyed in the Mercer Global Equality report indicated having WAGs and relying on them to carry out D\&I goals. Like many other D\&I interventions, the effectiveness of WAGs has not previously been examined. As the IT field continues to invest in programs such as WAGs to improve the participation of women in its workforce, it is pertinent to assess the D\&I interventions deployed in order to ensure their effectiveness. This paper utilizes the "Organizational Interventions Mitigating Individual Barriers" framework which examines the characteristics of WAGs in order to determine their potential opportunities and limitations to enhance participation of women in IT.
\end{abstract}

\section{Introduction}

Women make up only $26 \%$ of the information technology (IT) workforce [5]. This low representation is partially attributed to the fact that women tend to leave the IT workforce within the first five years at a rate of 56\%; twice that of men in IT and women in other fields [5], [19]. The high turnover is attributed to structural (e.g. occupational culture, institutional structures, and "masculine" promotion criteria) and social (e.g. stereotyping, questions of legitimacy, isolation, and work life balance) barriers (e.g., [2], [5], [32]) that leave women frustrated and cause them to leave IT at high rates.

To benefit from attracting and retaining women [5], [35], organizations often attempt to improve women's experiences in the workplace by implementing diversity and inclusion (D\&I) programs (referred to hereafter as interventions) [27], [40]. According to Forbes Insights [15], 97\% of companies surveyed had implemented D\&I interventions. Increasingly, organizations rely on affinity groups (AGs) to carry out D\&I strategies [20],

\author{
Mina Tari \\ The Information School, University of Washington \\ minatari@uw.edu
}

[24]. Goode's [20] study of AGs found that more than $70 \%$ of organizations that support AGs relied on them to partner with D\&I leaders to carry out diversity goals. What is especially important to note is that Women AGs (WAGs) are the most prevalent D\&I method in IT. The Mercer report [24] indicated that 93\% of companies had a WAG. The past few years has seen a resurgence of interest in AGs that the Mercer Global Equality report [24] attributes to millennial interest in workplace networking [24] [41]. In the research teams' conversations with D\&I personnel in major tech companies, they all emphasized the importance of WAGs. Surprisingly, WAGs, like many other D\&I interventions have not been sufficiently studied or assessed [41], [42]. Furthermore, the limited previous research on AGs lacks any theoretical grounding in their analyses [43].

The limited research on WAGs in light of its pervasiveness in IT raises question regarding the characteristics of WAGs and their suitability to carry out D\&I interventions in IT. Therefore, we ask the following research questions:

1. What are the characteristics of WAGs in the IT workplace?

2. Are WAGs suitably designed to address the barriers women experience in IT?

The goal of this paper is to offer a conceptual analysis of WAGs to assess their capacity and limitations to mitigate barriers women experience in IT and propose a research agenda to investigate the most pressing issues related to WAGs in IT. We focus on WAGs that are women-focused, rather than intersectional identities (e.g., African-American Women Affinity Groups) in this paper. We use the "Organizational Interventions Mitigating Individual Barriers" theoretical framework developed by Annabi and Lebovitz [3], [4] which provides us a holistic multilevel lens to conduct our analysis.

In the remainder of this paper we review the literature on AGs in general and WAGs in particular. Then we review the OIMIB and explain its utility for our analysis of WAGs, followed by our analysis of WAGs' opportunities and limitations. We conclude by offering a research agenda to explore the most salient issues to be explored. 


\section{What are Affinity Groups (AGs)?}

AGs, also known as employee resource groups (ERGs), network groups, or business resource groups (BRGs) are voluntary employee groups within organizations that support, develop, and advance employees [18], [22]. In their initial form, AGs are communities of employees that share common individual characteristics such as gender, race, ethnicity, sexual orientation, religion, or nationality [14], [43]. AGs date back to the National Black Employees Caucus formed in 1970 at Xerox inspired by the 1964 race riot [14]. Xerox developed the caucus to support black employees and address issues of discrimination and inclusion [14]. More recently, AG form around a wider set of characteristics such as interests (e.g. sports, service), professional roles (e.g. administrators), or personal roles (e.g. moms, dads) [20], [43] to support employees and enhance their experience and engagement in the organization.

Contemporary AGs serve several purposes for organizations and their members. The main purpose of AGs, though, is to facilitate interaction and social engagement to provide support and address issues of common interest [12]. Welbourne and McLaughlin [42] identified three types of AGs: social-cause centered, professionally-centered, and attribute-centered. Socialcause focused AGs are formed to bring together employees who wish to support public and social good (e.g. environmental work, healthcare); this type of AGs fulfill employee's need of being part of something bigger than themselves [8], [29]. Furthermore, individuals who participate in such AGs develop a higher sense of identification with the $A G$ and the organization [42]. Professionally-centered AGs are formed by employees in similar professional role (e.g. designers, engineers, or administrative staff) in the organizations to support their professional development. Attribute-centered AGs are more traditional form of AG's that focus on personal characteristics or demographics (e.g. women, Latino, LGBT) and serve different D\&I goals [17], [18]. In this paper we focus on the latter type of AG, attribute-centered AG, as a key D\&I intervention.

Attribute-centered AGs, such as WAGs, continue to be a very common D\&I intervention to carry out diversity goals. The Mercer report [24] identified at least three specific ways in which AGs serve D\&I strategies including: 1) serve as focus groups to provide feedback for D\&I strategists, 2) implement specific strategies, such as mentoring and onboarding, and 3) act as agents of cultural change in the organization. Furthermore, attribute-centered AGs, such as WAGs, legitimize marginalized identities by raising awareness, creating a safe space for emotional support to negotiate identity through communication, and providing a platform to advocate for equity [23]. They often assist organizations in creating policies to support positive cultural change.

Despite the prevalence of attribute-centered affinity groups, such as WAGs, the literature provides minimal evidence of the actual impact of such groups. We know little about how to best design and utilize AGs in general and WAGs in particular to achieve these significant D\&I roles. We have limited understanding of the capacities and limitations of WAGs. More specifically, [we want to [understand]]:

1. What are the characteristics of WAGs in the IT workplace?

2. Are WAGs suitably designed to address the barriers women experience in IT?

To fully assess WAGs capacity and limitations to satisfy the D\&I roles outlined above, we use the "Organizational Interventions Mitigating Individual Barriers" (OIMIB) theoretical framework [3], [4]. This framework is grounded in Information Systems (IS) gender theory which 1) depicts the characteristics of interventions deployed in IT organizations (their catalysts and objectives, methods they employ, and assessments they carry out);2) determines explicitly the barriers they are designed to address; 3 ) assesses the efficacy of these interventions mitigating specific barriers considering the individual differences of women in IT; and 4) illuminate the interplay between barriers individual women experience, intervention characteristics, and women's informal methods to address those barriers. Therefore, the OIMIB framework provides a holistic approach to investigate D\&I interventions and include individual-, intervention, and organization-level constructs. This holistic multilevel system view enables us to better understand WAGs' capacity and limitations to address the diversity of women's needs and experiences in IT, within the context of organizational and intervention characteristics that often present systemic barriers to women's inclusion in IT. The emphasis on interventionand organization-level constructs and their impact on individual-level constructs enables us to address these systemic issues inherent in the IT workplace that are causing women to leave.

\section{Organizational Interventions Mitigating Individual Barriers (OIMIB) Framework}

The OIMIB framework is comprised of four components: 1) individual women's experiences; 2) barriers present in the IT workplace; 3) organizational interventions; and 4) individual coping methods. 
The first component of the OIMIB framework, "Individual Women's Experiences" is grounded in the individual differences theory of gender in IT (IDTGIT) which emphasizes that women experience barriers present in the IT workplace on the individual level [33], [34]. An individual differences lens affords us a deeper understanding of the complexities of women's experiences and responses to barriers facing them [27]. Gaining a more nuanced understanding of how women experience barriers is necessary to illuminate how WAGs may address the barriers women face and improve their retention in the field. For this conceptual analysis of WAGs, we will not assess the first component of the OIMIB framework, "Individual Women's Experiences". This component of the model will be used in the empirical phase of the research. For details on IDTGIT please refer to Trauth et al., [37].

The second component of OIMIB, "Barriers Present in the IT Workplace," is an organizational-level construct. This component includes the effects of barriers first identified in Ahuja's [2] model. Societal and organizational structures create barriers for women in the IT workplace. Women in IT are subject to stereotyping that leads to mistaken assumptions about a woman's interests, capabilities, and skills, and push women toward typically "feminine" and less technical roles [1], [16], [28]. Stereotyping also manifests in "access and legitimacy" perceptions where women are viewed as intrinsically less capable than men [45] and their performance is evaluated differently from their male peers, causing them to be passed over for promotions due to assumptions about being "family focused" and "unwilling to travel" [21], [32]. Research has widely recognized the "double burden" women face when balancing work and family [6], [31]. While this barrier is not unique to IT women, specific qualities in IT make the work-life balance particularly challenging and tightly correlated with high turnover rates [2], [31]. Lastly, the combination of being a minority and often having poor supervisory relationships leaves women in IT feeling socially isolated for three reasons: 1 ) a lack of mentors, 2) limited successful role models, and 3) a limited professional network [2], [25], [32], [36]. For details on the effects of barriers on women in IT please refer to Annabi and Lebovitz [3], [4].

The third component of the OIMIB framework, "Individual Coping Methods," is an individual-level construct focusing on how women exercise agency and deploy individual coping methods to respond to barriers. These methods range from developing informal networks, seeking informal mentors, and ignoring barriers, to more severe responses, such as changing personal characteristics or leaving IT. Like the "Individual Women's Experiences" component, we will not consider this component in this conceptual analysis and reserve it for the empirical analysis.

The fourth and final component of the OIMIB framework, "Organizational Interventions," is an intervention-level construct. This includes the characteristics of organizational interventions for preventing, mitigating, and eliminating the barriers women experience, as identified in Table 1. Intervention characteristics influence the extent to which barriers exist and the level at which they negatively impact women. Annabi and Lebovitz [3] organized intervention characteristics into three categories: intervention catalysts, methods and practices deployed, and measurement processes. The intervention catalysts determine the organization's commitment level and, in turn, how effectively these interventions will address barriers. The catalysts influence whether, and to what extent, women utilize these interventions. The diverse methods and practices deployed in interventions are directly related to any intervention's effectiveness in addressing barriers. If intervention methods are successfully designed and deployed to address the barriers women experience, the barriers are eliminated. Lastly, measurement characteristics relate to the effectiveness of interventions at addressing barriers, creating accountability for the organization's specific catalysts and goals. Please refer to table 1 for details of organizational intervention characteristics.

To guide our analysis of WAGs, we utilize the "Organizational Interventions Characteristics" and "Effects of Barriers" components of the OIMIB framework. We use the two components at this conceptual stage in our research because it provides theoretically grounded constructs that illustrate the effects of barriers women experience, as well as the interventions intended to address barriers. In other words, these two components of OIMIB define: 1 ) the range of problems that interventions need to solve "Barriers in the IT Workplace"; and 2) the nature of the solutions designed and deployed to address the problems of "Organizational Interventions Characteristics." Craig [13] specifies that analysis and assessment of interventions in light of the problems they are designed to address is essential to determine the effectiveness of D\&I interventions.

\section{The Characteristics of WAGs}

In this section, we utilize the OIMIB framework to analyze the characteristics of WAGs and assess their effectiveness in addressing the barriers they are expected to address. 


\subsection{WAGs catalysts and objectives}

The main catalysts for WAGs are the need to: 1) support traditionally marginalized employees (those historically excluded from mainstream organizational processes and decision making based on gender, race, ability, etc.) and 2) address issues of discrimination and inclusion in organizations [14]. Since the 1970's, when the first AGs emerged, organizational diversity efforts evolved from merely valuing diversity (recognizing and appreciating differences) to managing diversity (implementing programs to recruit and retain a diverse workforce) [44]. This shift has been supported by a growing business case for diversity and D\&I interventions. For WAGs, the business case is based on the group's positive impact on recruitment and their ability to provide support for women and improve retention and advancement. Potential employees from marginalized populations have come to expect that companies have D\&I interventions like WAGs and are attracted to companies that have a reputation for social responsibility [12]. Furthermore, research suggests that retaining women increases market share, productivity, creativity, problem solving ability, and innovation, which ties diversity management to a company's bottom line [3], [42], [44].

Although WAGs hold significant potential benefits for organizations, our understanding remains conceptual and not empirical. The catalysts described above present a compelling theoretical case for WAGs to flourish and improve the retention of women in IT, yet we know little about how these catalysts and objectives take shape in reality and how these catalysts interact with barriers women experience in IT and affect those experiences. Utilizing OIMIB, which is grounded in intersectional, inclusive theory of gender in IT, we will guide empirical efforts to enhance our understanding of WAGs and their effectiveness to mitigate and eradicate barriers women experience.

Table 1. OIMIB Intervention Characteristics

\begin{tabular}{|l|}
\hline Catalysts and Objectives \\
\hline Enhance corporate social responsibility \\
\hline Reduce turnover costs \\
\hline Raise awareness and encourage women \\
\hline Develop reputation and culture for being supportive \\
\hline Enhance innovation and team performance \\
\hline Improved financial business performance \\
\hline Better reflection of and service to customers \\
\hline
\end{tabular}

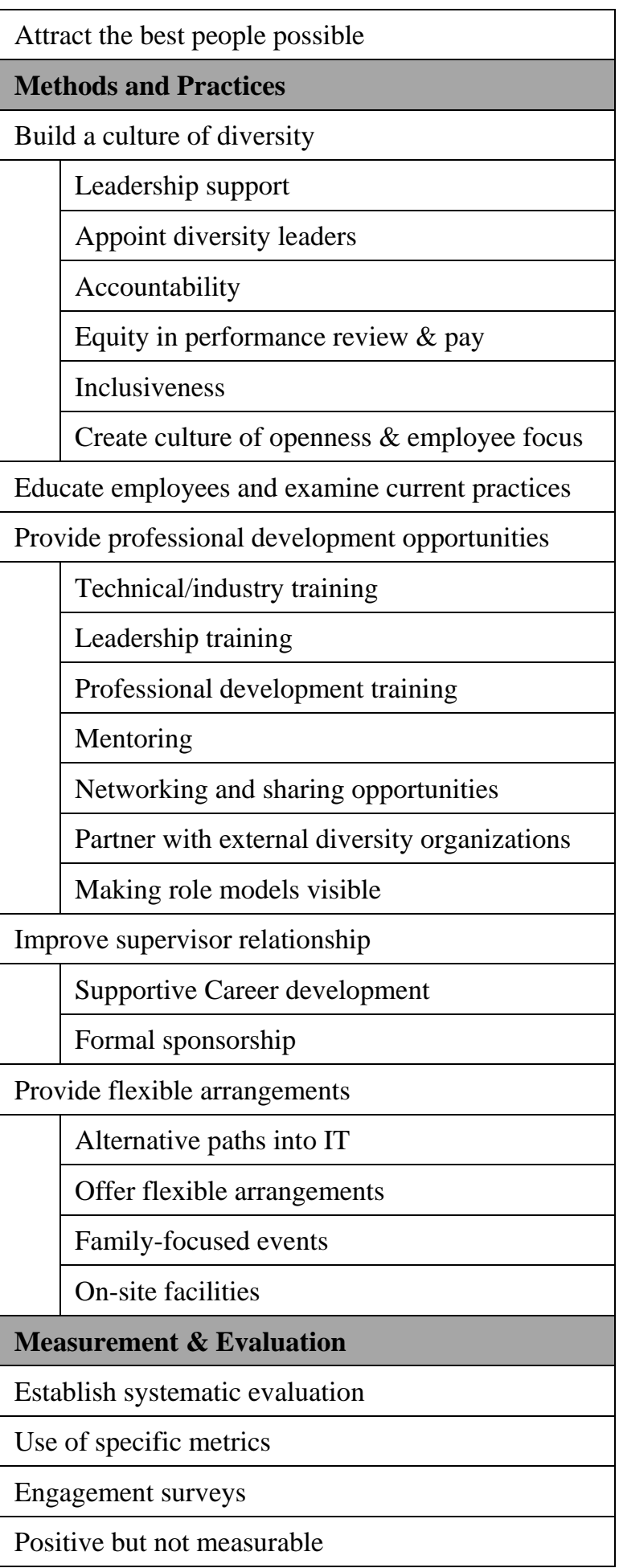

\subsection{WAGs methods}

OIMIB posits building a culture of diversity as a key characteristic of effective interventions. Organizations 
build such a culture by providing leadership support, formal initiative leaders, and accountability among other things. WAGs exist on a spectrum: from grassroots efforts to company-initiated and supported groups that demonstrate organizational commitment to create a culture of diversity and inclusion [8]. As WAGs have become more popular, organizations are more prone to providing the groups with a variety of structural support. While participation in the groups is voluntary, WAGs are sponsored and provided resources by their organizations, the extent of which can vary from setting up an email listserv to appointing senior leadership as champions for guidance [14], [43], [44]. WAGs also tend to have formal governance and structure, with support from senior executives, steering committees, and managerial liaisons [14], [43]. Currently as AGs (including WAGs) have become more popular, the average support for them is " $\$ 7,203$ for every $100 \mathrm{ERG}$ members, and many companies spend well into six figures every year (not counting the cost of technology, facilities, staff support, and other non-financial resources provided to the groups)" [9]. The more financial and leadership support WAGs secure, the more likely they are to be effective.

On an organizational level, WAGs can help create a culture of diversity and inclusion. The groups can also improve informal organizational processes of both startups and established companies [38]. WAGs offer opportunities for bridging cultural differences across company boundaries, connecting with the wider community, and providing varying and innovative perspectives [43]. WAGs have been shown to improve communication between employees, creating opportunities to share information in informal channels and decision-making systems, and establishing paths for advocacy [14], [38], [43]. The groups can also improve informal organizational processes of both start-ups and established companies [38]. WAGs also help build affiliation within the community, improving collaboration, conflict-management, and internal loyalties [14], [43]. However, the existing research is not clear on how this impact improves inclusion in work environment for women and how that might affect their retention. Therefore, we conclude that the existing literature is lacking in the assessment and evaluation of WAGs

The most common intervention methods identified in OIMIB and evident in the WAGs offer members professional development and networking opportunities, such as formal and informal mentoring programs, hosting educational workshops and guest speakers, and improving access and visibility to senior leadership [14], [43], [44]. In addition to the professional development, WAG members benefit on a personal level from having a more meaningful sense of work and community to improve commitment to their organization [43]. Even though WAGs can require working more hours, employees have reported that the experience is energizing [43].

The OIMIB framework identified providing flexible work arrangements and improving supervisory relationships as two other important methods to employee in organizations to address barriers women face. Understandably, WAGs do not formally or specifically address these two key methods. However, WAGs often champion flexible work arrangements and supervisory relationship issues by providing feedback and serving as focus groups to the leadership of the organization tasked with such efforts [24].

\subsection{Assessment and evaluations: impact on retention and advancement of women}

Depending on a commitment to truly inclusive practices and sufficient knowledge in how to facilitate a better understanding of the complexities of barriers women face in IT, WAGs can have positive or negative effects on retention and advancement. However, as the literature provides little to no evidence of theoretical grounding or systematic assessment of WAG outcomes in relation to barriers, it is difficult to identify if WAGs are achieving D\&I goals, or damaging the situation at their organizations. Therefore, assessment of intervention outcomes to assess efficacy of WAGs at addressing barriers provides critical accountability for the organization's specific catalysts and goals [3], [13].

\subsection{How do WAGs address barriers women experience?}

WAGs provide spaces for marginalized identities to combat isolation by feeling social and business inclusion [30], [44]. These groups can increase morale, retention, and inclusion of women [30], [39]. Furthermore, WAGs provide a space for women across different level and functions of the organization to come together [43]. Facilitating such networking opportunities gives women access to information and opportunities they would not otherwise have.

WAGs may also be a catalyst for valuing diverse employees' insights, experiences, and skills as a resource for learning and change which organizations can use to better influence their culture, rather than only looking to gain diversity for access and legitimacy in a market [39]. This addresses the legitimacy barrier women often experience. What is not clear however, is how the WAGs transform the general environment in the organization to be more inclusive to women beyond the group. 


\subsection{What are the limitations of WAGs that impede their effectiveness to address barriers women experience?}

Most WAG efforts in the literature focus on the women, by providing much appreciated educational, professional, and networking opportunities [3]. However, these efforts focus on the marginalized individual and do not address the barriers of stereotyping, access and legitimacy, and isolation women experience in IT on a regular basis due to their interactions with their colleagues.

More significantly, if WAGs are not valued and supported by the organization at large, their impact will diminish within an organization. This requires organizational leadership to understand the structural and systemic issues of oppression and effect on marginalized identities.

WAGs, being voluntary, can be more easily subverted by managerial staff or used to spread messages of diversity without any significant action (e.g., promoting how friendly the company is to gender diversity while not having women in leadership roles). For example, Williams, Kilanski, \& Muller [44] observed D\&I initiatives in a large oil and gas companies, and found that a grassroots network group on work-family balance was institutionalized after receiving support from top managers. Though it seemed successful, the group did not require resources or challenge the company's limited parental support allowing the company to maintain "the illusion of corporate fairness and support for diversity" [44]. Additionally, the authors found that while participants enjoyed the social and emotional support of discussion, there were doubts about the impact on professional development [44]. As that particular instance, WAGs lacked participation from those in leadership roles, it followed that networking and professional development was limited.

As with most groups and communities, it can take time for WAGs to achieve their goals effectively, depending on frequency of meetings and commitment of members and leaders [38]. Welbourne, Rolf, \& Schlachter [43] discuss how the longer WAGs exist, the higher the potential to shift their focus to more sociallydriven gatherings than professionally-driven, losing the incentive to incite organizational change [43]. Furthermore WAGs may face backlash from those not in the group regarding funding, or perceived discrimination [43]. Therefore, any organization must be able to clearly and effectively communicate the objectives of WAG in order to realize their potential benefits Again, leadership support and championship are especially important to set the tone and culture at the organization at large.

Employees may be hesitant to join WAGs depending on their perceptions of the group's objectives and fear from dominant group's reaction. If women perceive the group as too radical or too combative, they may be reluctant to have those traits associated with them [18]. Many may also not have any interest or connection with the goals, which touches upon a larger issue of lack of empathy or affiliation to the specific identity of women being presented by the group. As WAGs are intended to cross hierarchical lines, Welbourne, Rolf, \& Schlachter [43] contemplate how dynamics can change between supervisor and employee in the context of a WAG. The concerns were the potential limitation of discussion for fear of consequence from a member of management ranks [11], [43].

The limitations we identified relating to the WAGs ability to support women, create a safe space for them to receive support and feel connected to others, and advocate and participate in creating positive cultural change to ensure equity are theoretical and anecdotal in the literature. Significant empirical effort is needed to investigate these limitations more fully.

\section{Proposed Research Agenda}

Our analysis of WAG characteristics and our assessment of how they address barriers women face in IT provides great insight on areas to capitalize on and areas to investigate. Most significantly, our review of the literature revealed that there is a significant need for theoretically grounded empirical research to better understand and assess the efficacy of WAGs, as well as ways to better support and utilize them for the retention of women in IT. The discussion below offers our research agenda.

\subsection{A need for theoretically grounded critical research on WAGs}

To improve the retention of women in IT, we must clearly and effectively address the barriers they experience. Our assessment of WAG characteristics revealed that these groups provide great benefits for their members. However, in their current state, WAGs are not currently positioned to address the barriers inherent in the power structures that privilege interests and experiences of the dominant culture that simultaneously silence or ignore marginalized IT women [23], [26]. Therefore our investigation of WAGs must be theoretically based and grounded in critical epistemology that will uncover these power dynamics and give voice to marginalized groups [23], [37]. Such 
empirical efforts must assess the perspectives of women, men, and other gender identities in order to develop a holistic understanding of the role WAGs play in organizations and how members of the organizations with different gender identities support or detract from WAGs ability to empower women and champion equity for all in the organization.

\subsection{Utilize OIMIB to investigate WAGs' impact on women}

Though the literature on AGs provides insight about the efficiency of certain characteristics, we have previously discussed its lack of holistic theoretically grounded understanding of organizational interventions aimed at women's participation in IT. OIMIB's multilevel holistic approach and theoretically grounded framework investigates the interplay between WAG characteristics, barriers, and individual differences which will inform the design, implementation, and assessment of WAGs. The framework will explain the implications of organizational level and WAG characteristics on the diversity of women participating in WAGs. This research effort will be guided by the following research questions:

1. What are the characteristics of WAGs in the IT workplace?

2. What are the obstacles in the design, deployment, and assessment of WAGs?

3. What is the nature of participation in and utilization of WAGs by IT women?

4. How effective are WAGs in addressing the barriers women face in the IT workplace?

5. How do nonparticipants perceive the need for and benefits of WAGs? What impact might these perceptions have on WAGs participants' participation and experiences?

6. What role do WAGs play in organization strategy for retention and advancement of women?

7. What type of leadership involvement best supports WAGs?

\subsection{Challenging institutional bias and building empathy}

Carter [10] and Blitz and Kohr Jr., [7] discuss how identity-based oppression shapes organization's structures, subsequently affecting organizational-level performance and individual behavior. By understanding how institutionalized oppression affect construction of barriers for women in IT, it is easier to create organizational structure to combat it. However this is particularly difficult as employees may not be aware of the systemic influences, and only focus on individual- level acts. Thus, organizations need to develop internal support systems to assist employee understanding [7]. WAGs can be one avenue for improving members understanding of complexities of oppression and ways to unravel and address them. Therefore, it is important to investigate the views of WAG members as well as non-members from marginalized and dominant groups . Our research agenda includes an investigation of the perceptions and attitudes non WAG participants hold of WAGs and women in order to reveal institutional bias against women and WAGs.

\subsection{WAGs within the system of AGs and D\&I interventions}

Lambertz-Berdnt [23] analyzed attribute-based AGs such as WAGs and made suggestions evaluating their effectiveness. They suggest that having experienced facilitators, resources and logistical space, and time during the workday to attend AGs increased the groups' positive impact. One of their major findings was that AGs without an intersectional discussion for visible and nonvisible identities lacked cohesiveness. Having cooperation and collaboration between AGs for those with multiple identities has the potential to build cultural integration and cultural agility [23], [41]. Therefore, we propose to use OIMIB to conduct a systematic assessment of WAGs and their relationship with other AGs and other interventions to address areas for highest impact to create more inclusive organizational culture.

\section{Conclusion}

In conclusion, we emphasize the importance of theoretically grounded research to study D\&I interventions and assess their effectiveness in improving the experiences of marginalized populations within IT. WAGs are the most common D\&I intervention in IT. This paper presented a conceptual analysis of WAGs that was theoretically grounded in the OIMIB framework. This framework illuminated the characteristics of WAGs and identified areas of strengths and limitations in their ability to address the barriers women in IT experience. The paper concluded with a set of proposed areas for future research. Pursuing empirical research that is grounded in theory that accounts for the individual differences of women as well as organizational and intervention characteristics will inform our understanding of WAGs and improve their design, implementation, and assessment. 


\section{References}

[1] Accenture, "Millennial women in the workplace success index: Striving for balance," Jan. 2010 [Online]. Available: http://www.accenture.com/us-

en/company/people/women/Pages/womens-research-2010-

millenial-women-summary.aspx. [Accessed: 23-Oct-2011].

[2] Ahuja, M., "Women in the information technology profession: A literature review, synthesis, and research agenda," European Journal of Information Systems, vol. 11, pp. 20-34, Mar. 2002

[3] Annabi, H. and S. Lebovitz, "Improving the Retention of Women in the IT Workforce: An Investigation of Gender Diversity Interventions," forthcoming, Information Systems Journal.

[4] Annabi, H. and S. Pels, "Understanding How Effective Are Diversity Interventions At Addressing the Barriers Women Face in the IT Workplace? A Theoretical Model," Proceedings of the 22nd Americas Conference on Information Systems, San Diego, CA. Aug. 2016

[5] Ashcraft, C., B. McLain, and E. Eger, Women in Tech: The Facts. Boulder, CO: National Center for Women and Information Technology, 2016.

[6] Blair-Loy, M., Competing Devotions: Career and Family among Women Executives. Oakland, CA: University of California Press, 2007

[7] Blitz, L. and B. Kohl Jr., "“Addressing Racism in the Organization: The Role of White Racial Affinity Groups in Creating Change," Administration in Social Work, vol. 36, pp. 479-498, Feb. 2012

[8] Briscoe, F. and S. Safford, "Employee Affinity Groups: Their Evolution from Social Movements Vehicle to Employer Strategies" Perspectives on Work, vol. 14, no. 1, pp. 42-45, Dec. 2011

[9] Brotherton, P., "Employee Resource Groups Still Going Strong," Association for Talent Development, Aug. 2011.

[10] Carter, R. T., Addressing cultural issues in organizations: Beyond the corporate context. R. T. Carter, Ed. Thousand Oaks, CA: Sage Publications, 2000

[11] Connelly, C. and E. K. Kelloway, "Predictors of employees' perceptions of knowledge sharing culture," Leadership \& Organization Development Journal, vol. 24, no. 5, pp. 294-391, Feb. 2003

[12] Cox, T., and S. Blake, "Managing cultural diversity: Implications for organizational competitiveness," Academy of Management Executive, vol. 5, pp. 45-56, Aug. 1991

[13] Craig, A, "Theorizing about gender and computing interventions through an evaluation framework," Information Systems Journal, vol. 26, no. 6, pp. 585-611, Apr. 2016
[14] Douglas, P. H., “Affinity groups: Catalyst for inclusive organizations," Employment Relations Today, vol. 34, pp. 1118, Jan. 2008

[15] Forbes Insight, "Global Diversity and Inclusion: Fostering Innovation through a Diverse Workforce," New York, NY: Forbes Insight, 2011

[16] Foust-Cummings, H., N. Carter, and L. Sabattini, Women in Technology: Maximizing Talent, Minimizing Barriers, New York, NY: Catalyst, 2008

[17] Friedman R. A. and B. Holtom, "The effects of network groups on minority employee turnover intentions," Hum. Resour. Manage., vol. 41, no. 4, pp. 405-421, Dec. 2002.

[18] Friedman, R., and K. Craig "Predicting Joining and Participating in Minority Employee Network Groups," Industrial Relations, vol. 43, no. 4, pp. 793-816, Oct. 2004

[19] Glass, J., et al., "What's so special about stem? A comparison of women's retention in stem and professional occupations," Social Forces, vol. 92, pp. 723-756, Aug. 2013

[20] Goode, S., "Are Employee Resource Groups Good for Business?” HR Magazine, pp. 24-25, Sep. 2016

[21] Hewlett, S., et al., The Athena factor: Reversing the brain drain in science, engineering, and technology, Boston, MA: Harvard Business School Publishing, 2008.

[22] Kaplan, M., M., E. Sabin, and S. Smaller-Swift, "The Catalyst Guide to Employee Resource Groups, 1-Introduction to ERGS," Catalyst Knowledge Center, Sep. 2009.

[23] Lambertz-Berndt, M., "Communicating Identity in the Workplace and Affinity Group Spaces" Studies in Media and Communication, vol. 4, no. 2, pp. 110-124, Oct. 2016

[24] Mercer Inc., "ERGs come of age: The evolution of employee resource groups," 2011. [Online]. Available: http://www.orcnetworks.com/system/files/story/2011/5849/er gs_come_of_age_2011_study_pdf_30909.pdf. [Accessed 19Nov-2013].

[25] Orser, B., et al., "Gender challenges of women in the Canadian advanced technology sector" CATAWIT Forum: Women in Technology, pp. 1-22, Nov. 2007

[26] Parker P., "Negotiating identity in raced and gendered workplace interactions: The use of strategic communication by African American women senior executives within dominant culture organizations," Communication Quarterly, vol. 50 no. 3-4, pp. 251-268, May 2002

[27] Quesenberry, J. and E. Trauth, "The (dis)placement of women in the IT workforce: An investigation of individual career values and organisational interventions," Information Systems Journal, vol. 22, pp. 457-473, Jun. 2012 
[28] Scott-Dixon, K., Doing IT: Women Working in Information Technology. Toronto, Ontario, Canada: Sumach Press, 2004

[29] Scully, M. and A. Segal, "Passion with an umbrella: Grassroots activists in the workplace," Social Structure and Organizations Revisited, vol. 19, pp. 125-168, May 2002.

[30] Segal, S., 2013. “Affinity Group Danger Zones,” HR Magazine, pp. 75-90, 2013

[31] Shafer, P. and B. Trautlein, "Women in Technology: 2007 Report,” WITI SAVVY, pp. 19-23, May 2007

[32] Simard, C., et al., Climbing the Technical Ladder: Obstacles and Solutions for Mid-Level Women in Technology. Palo Alto, CA: Stanford University and Anita Borg Institute for Women and Technology, 2008

[33] Trauth, E., "Odd girl out: An individual differences perspective on women in the IT profession," Information Technology and People, vol. 15, pp. 98-118, Jun. 2002

[34] Trauth, E., "Theorizing gender and information technology research" In: Encyclopedia of Gender and Information Technology, E. Trauth, Ed. Hershey, PA: Idea Group Publishing, 2006, pp. 1154-1159.

[35] Trauth, E., "Rethinking gender and MIS for the twentyfirst century" In: The Oxford Handbook on MIS, R. Galliers, and W. Currie, Eds. Oxford, U.K.: Oxford University Press, 2011

[36] Trauth, E., et al., "Investigating diversity in the global IT workforce: An analytical framework" In: Human Resource Management of IT Professionals, F. Niederman, and T. Ferratt, Eds., Charlotte, NC: Information Age Publishing, 2006, pp. 331-360.

[37] Trauth, E., J. Quesenberry, and H. Huang, "Retaining women in the US IT workforce: Theorizing the influence of organizational factors," European Journal of Information Systems, vol. 18, pp. 476-497, Oct. 2009

[38] Van Aken, E. M., D. G. Monetta, and D. S. Sink "Affinity groups: The missing link in employee involvement," Organizational Dynamics, vol. 22, no. 4, pp. 38-54, Mar. 1994

[39] Vohra, N., and V. Chari "Inclusive Workplaces: Lessons from Theory and Practice," Vikalpa: The Journal for Decision Makers, vol. 40, no. 3, pp. 324-362, Sep. 2015

[40] von Hellens, L., E. Trauth, and J. Fisher, "Increasing the representation of women in the information technology professions: Research on interventions," Information Systems Journal, vol. 22 pp. 343-353, Aug. 2012

[41] Ward, G., "An Investigation Of Performance And Participation In Employee Resource Groups At A Global Technology Company," Ph.D. dissertation, Dept. of
Instructional Technology, Wayne State University, Detroit: MI, 2012

[42] Welbourne, T. M. and L. L. McLaughlin, "Making the Business Case for Employee Resource Groups," Employment Relations Today, pp. 35-43, Jul. 2013

[43] Welbourne, T. M., S. Rolf, and S. Schlachter, “"Employee Resource Groups: An Introduction, Review and Research Agenda'," Academy of Management Proceedings, vol. 2015, no. 1, pp. 15661-15661, Sep. 2015.Welbourne et al., 2013

[44] Williams, C., K. Kilanski, and C. Muller, "Corporate Diversity Programs and Gender Inequality in the Oil and Gas Industry," Work and Occupations vol. 41, no. 4, pp. 440-476, Nov. 2014

[45] Wilson, M., "A conceptual framework for studying gender in information systems research," Journal of Information Technology, vol. 19, pp. 81-92, Mar. 2004 